\title{
Cardiac tamponade associated with delayed ascending aortic perforation after blunt chest trauma: a case report
}

\author{
Dae Woong Ryu* and Mi Kyung Lee
}

\begin{abstract}
Background: Cardiac tamponade due to aortic injury after blunt trauma is a rare and potentially fatal injury. Most aortic injuries caused by blunt trauma present as aortic dissection or rupture of the aortic isthmus. Several cases of delayed aortic injury have been reported. However, all of these injuries were observed in the descending aorta because they had been caused by a posterior rib fracture.

Case presentation: We report the first case of cardiac tamponade associated with delayed ascending aortic perforation 2 weeks after blunt trauma. The patient was an 81-year-old man.

Conclusion: In cases of blunt chest trauma, delayed ascending aortic injury causing cardiac tamponade is possible associated with various causes such as direct injury by fractured rib or delayed aortic perforation of initial blunt injury.
\end{abstract}

Keywords: Cardiac tamponade, Delayed aortic injury, Rib fracture, Blunt chest trauma

\section{Background}

Cardiac tamponade after blunt trauma is rare and usually associated with rupture of cardiac chambers on the relatively weaker right side [1]. Aortic injury causing tamponade after blunt trauma is an even rarer catastrophic injury that leads to death. Blunt trauma can inflict injury to the aorta through several mechanisms. Most aortic injuries caused by blunt trauma present as aortic dissection or rupture of the aortic isthmus due to indirect forces. Direct injury of the aorta can sometimes be caused by a fractured bone fragment [2]. Several cases of delayed aortic injury caused by rib fracture have been reported. However, almost all cases involved the descending aorta owing to a posterior rib fracture. In our case, a laceration-type wound approximately $7 \mathrm{~mm}$ in size with $2 \mathrm{~mm}$ perforation (Fig. 2) was found at the level of perforated pericardium (Fig. 1a). To our knowledge, delayed ascending aortic perforation after blunt trauma has not been reported. We describe a case of cardiac tamponade associated with delayed ascending aortic perforation 2 weeks after blunt trauma.

\footnotetext{
* Correspondence: bart21@hanmail.net

Department of Thoracic and Cardiovascular Surgery, School of Medicine, Wonkwang University, 460 Iksandae-ro, Iksan, Jeonbuk 570-749, Republic of Korea
}

(c) The Author(s). 2017 Open Access This article is distributed under the terms of the Creative Commons Attribution 4.0 International License (http://creativecommons.org/licenses/by/4.0/), which permits unrestricted use, distribution, and reproduction in any medium, provided you give appropriate credit to the original author(s) and the source, provide a link to the Creative Commons license, and indicate if changes were made. The Creative Commons Public Domain Dedication waiver (http://creativecommons.org/publicdomain/zero/1.0/) applies to the data made available in this article, unless otherwise stated.

\section{Case presentation}

An 81-year-old man presented to our hospital with chest pain and dyspnea 2 weeks after sustaining blunt trauma from a cultivator accident. At the time of the accident, he did not seek medical care. On admission, he was alert and complained of chest pain. He had a history of hypertension and hyperlipidemia which was being treated with medications for 9 years. A bruise was noted on the anterior chest. His blood pressure had decreased to $70 / 50 \mathrm{mmHg}$; however, his other vital signs were normal.

Laboratory tests revealed decreased hemoglobin $(8.5 \mathrm{~g} / \mathrm{dL})$, elevated plasma lactate $(104 \mathrm{mg} / \mathrm{dL})$, and normal creatine kinase (CK), CK-MB, and troponin $\mathrm{T}$ levels. The other laboratory values were within the reference limits.

An electrocardiogram indicated sinus rhythm with premature atrial complexes and low voltage QRS complexes. Initial contrast-enhanced computed tomography (CT) showed a collection of hemorrhagic fluid in the pericardium and suspected leakage of contrast medium on the right ventricle (Fig. 1b), with multiple fractured ribs(3rd to 5 th ribs) on the right aspect of the chest. The ascending aorta was measured to be about $45 \mathrm{~mm}$ in maximum diameter (Fig. 1a). 


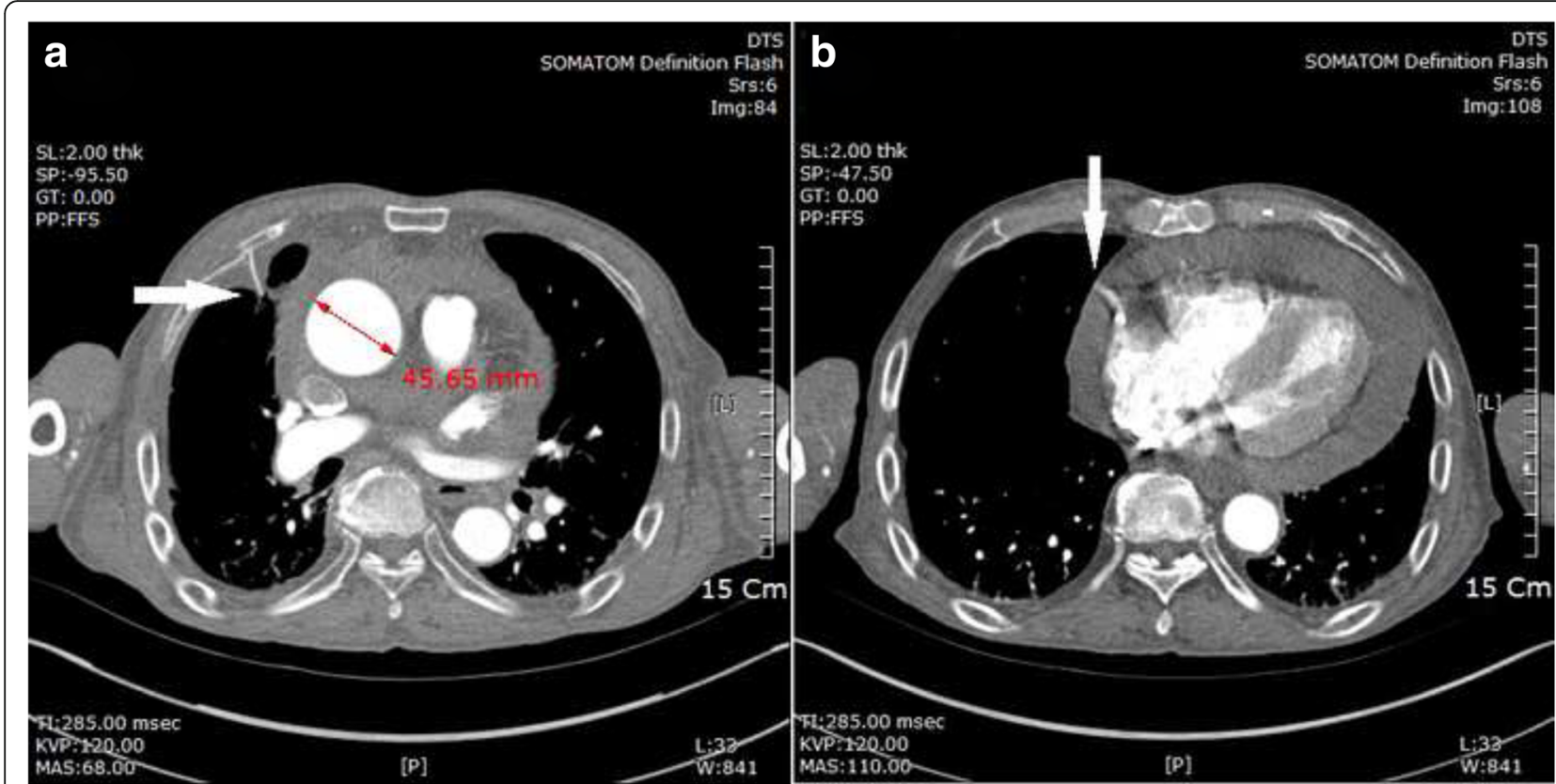

Fig. 1 a Bony fragment of the fractured 4th rib (arrow). The ascending aorta was measured to be about 45 mm in maximum diameter. b Contrast-enhanced computed tomography showing collection of hemorrhagic fluid in the pericardium, with enhancement in the right side of the heart, suggesting leakage of contrast medium (arrow)

We suspected right-sided cardiac rupture, established an anesthetic plan based on the cardiac rupture and performed emergency surgery. After median sternotomy, immediately after pericardial incision, approximately $200 \mathrm{~mL}$ stagnated blood gushed out and the blood pressure normalized. After removing the hematoma covering the heart, continuous pulsatile flow of fresh blood was observed coming from the ascending aorta. A lacerationtype wound approximately $7 \mathrm{~mm}$ in size with $2 \mathrm{~mm}$ perforation (Fig. 2) was closed with a 4-0 pledgeted polypropylene suture. We could not find any obvious laceration of the parietal pericardium except for a small

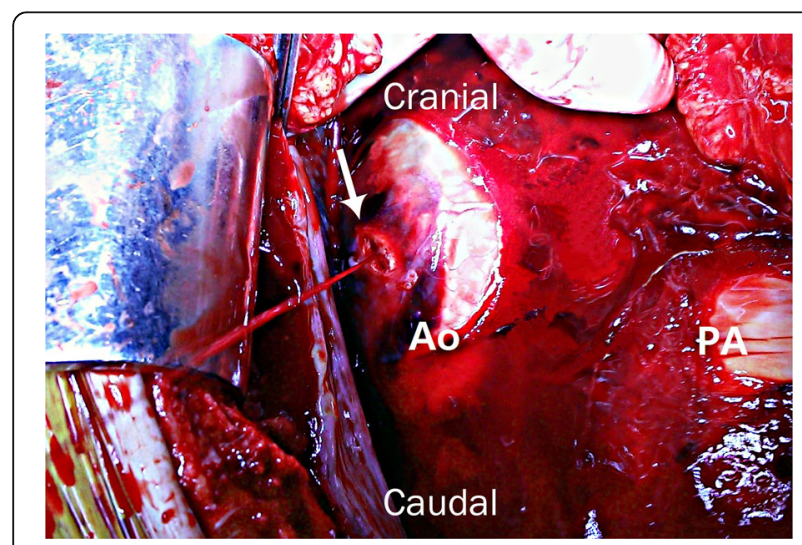

Fig. 2 A laceration-type wound approximately $7 \mathrm{~mm}$ in size with $2 \mathrm{~mm}$ perforation on the right side of the ascending aorta was found at the level of perforated pericardium (arrow) perforation and an abrasion at the level of the ascending aortic injury. The tip of sharp bony fragment of the fractured 4th rib was removed using a rongeur. The patient was discharged after 14 days, without any complications.

\section{Discussion}

Cardiac tamponade after blunt trauma is rare and associated with very high mortality. The main cause of tamponade is rupture of the cardiac chambers, predominantly on the relatively weaker right side of the heart [3]. However, cardiac tamponade caused by aortic injury after blunt trauma is extremely rare [1]. In our case, CT showed a collection of hemorrhagic fluid in the pericardium, suspected leakage of contrast medium from the right heart, and fractured ribs sufficiently distant from the heart (Fig. 1). Therefore, we initially assumed that delayed cardiac tamponade was caused by rupture in the right side of the heart.

Aortic injury after blunt trauma usually occurs in the extrapericardial part of the aorta, and only $15 \%$ of patients with an aortic injury after blunt trauma survive until hospital arrival. The mechanism of injury remains the most important factor, e.g., falls from $>10 \mathrm{ft}$., motor vehicle accidents at speeds $>30 \mathrm{mph}$, unrestrained drivers, ejected passengers, and pedestrians struck by motor vehicles [4].

Blunt trauma can inflict injury to the aorta through several mechanisms. The aorta can be damaged by indirect forces such as stretching, shearing due to deceleration loads, or increases in internal blood pressure. 
In such cases, the aortic isthmus is the most common site of rupture. A displaced, fractured thoracic vertebra, or bony intrusion by a fractured rib and clavicle, can cause direct injury to the aorta [2]

Multiple rib fractures are common in blunt chest trauma, and can lead to various injuries of intrathoracic organs such as pneumothorax, hemothorax, lung contusions, and injuries to neighboring organs [5]. A great vessel injury caused by a rib fracture can be fatal but, fortunately, seldom occurs [6].

However, several cases of delayed aortic injury caused by a rib fracture have been reported, and almost all patients had involvement of the descending aorta owing to a posterior rib fracture [5]. Because the ascending aorta is protected by the sternum and the ribs are relatively distant from the heart, as in our case, ascending aortic injury after blunt trauma is rarely reported in the literature. The cause of such delayed perforation is uncertain; however, we suggest that superficial injury of the aorta associated with a bony fragment or direct blunt injury occurred at the time of the accident but perforation was delayed. Continuous exposure to high pressure in the aorta may have led to stretching of the wound, and perforation eventually occurred 2 weeks later [7]. Alternatively, the physical movements of the patient might have caused a new laceration from a bony fragment [8].

If delayed aortic perforation had initiated from the intimal tear, arterial blood pressure would have forced blood between the layers of the aortic wall forming a false aneurysm [2]. However, in our case, false aneurysm was not found in CT scan. This suggests that the injury began from outside the aorta associated with a fractured rib or direct blunt injury.

Because the patient did not seek medical care at the time of the accident, we were not able to determine the exact cause. However, if physical movements caused a new laceration after the accident, we believe that removal of a bony fragment by means of video-assisted thoracoscopic surgery (VATS) could have prevented the perforation, as reported by Funaki et al. [9].

\section{Conclusion}

Even in cases of blunt chest trauma without a fatal injury, delayed ascending aortic injury causing cardiac tamponade is possible associated with various causes such as direct injury by fractured rib or delayed aortic perforation of initial blunt injury. Therefore, careful follow-up is needed, and if periaortic bony fragments pose risks to the aorta, active preventive management such as rib resection by using VATS or thoracotomy may be able to prevent fatal outcomes.

\section{Abbreviations}

CK: Creatine kinase; CT: Computed tomography; VATS: Video-assisted thoracoscopic surgery
Acknowledgments

The authors appreciate the patient's consent to this case report.

Funding

This paper was supported by a grant from Wonkwang University in 2016.

Availability of data and materials

The datasets supporting the conclusions of this article are included within the article.

Authors' contributions

DR and ML performed the operation. DR drafted the manuscript. Both authors read and approved the final manuscript.

Competing interests

The authors declare that they have no competing interests.

Consent for publication

Written informed consent was obtained from the patient for the publication of this case report and any accompanying images. A copy of the written consent is available for review by the Editor of this journal.

Ethics approval and consent to participate

Not applicable.

Received: 29 November 2016 Accepted: 8 June 2017

Published online: 17 June 2017

References

1. Brathwaite CE, Rodriguez A, Turney SZ, Dunham CM, Cowley R. Blunt traumatic cardiac rupture: a 5-year experience. Ann Surg. 1990;212:701-4.

2. Richens $D$, Field $M$, Neale M, Oakley C. The mechanism of injury in blunt traumatic rupture of the aorta. Eur J Cardiothorac Surg. 2002;21:288-93.

3. Leavitt BJ, Meyer JA, Morton JR, Clark DE, Herbert WE, Hiebert CA. Survival following nonpenetrating traumatic rupture of cardiac chambers. Ann Thorac Surg. 1987;44:532-5.

4. O'Conor CE. Diagnosing traumatic rupture of the thoracic aorta in the emergency department. Emerg Med J. 2004;21:414-9.

5. Boyles AD, Taylor BC, Ferrel JR. Posterior rib fractures as a cause of delayed aortic injury: a case series and literature review. Inj Extra. 2013;44:43-5.

6. Morimoto $Y$, Sugimoto T, Sakahira H, Matsuoka H, Yoshioka Y, Arase H. Successful management of threatened aortic rupture late after rib fracture caused by blunt chest trauma. Ann Vasc Surg. 2014;28:1035e11-3.

7. Yang X, Xia L, Pan K. Delayed aortic rupture following perforating trauma. Ann Transl Med. 2014;2:102.

8. Kigawa I, Fukuda I, Fujii Y, Yamabuki K. A sharp edge of the fractured ribs caused the aortic injury at body-position change: a case report. Nihon Kyobu Geka Gakkai Zasshi. 1992:40:1116-20.

9. Funaki S, Inoue M, Minami M, Okumura M. Video-assisted thoracoscopic resection of fractured ribs to prevent descending aorta injury in patient with chest trauma. Ann Thorac Cardiovasc Surg. 2014;20:173-4.

Submit your next manuscript to BioMed Central and we will help you at every step:

- We accept pre-submission inquiries

- Our selector tool helps you to find the most relevant journal

- We provide round the clock customer support

- Convenient online submission

- Thorough peer review

- Inclusion in PubMed and all major indexing services

- Maximum visibility for your research

Submit your manuscript at www.biomedcentral.com/submit

) Biomed Central 